\title{
Author Correction: Structure of severe fever with thrombocytopenia syndrome virus L protein elucidates the mechanisms of viral transcription initiation
}

Panpan Wang, Lu Liu, Aijun Liu, Liming Yan, Yong He, Shu Shen, Mingxu Hu, Yu Guo, Haiguang Liu (D), Chuang Liu, Yinying Lu, Peiyi Wang, Fei Deng (D), Zihe Rao D and Zhiyong Lou (D)

Correction to: Nature Microbiology https://doi.org/10.1038/s41564-020-0712-2, published online 27 April 2020.

In the version of this Article originally published, there were errors in the main text and in Fig. $2 \mathrm{~d}-\mathrm{f}$ owing to the misplacement of residues spanning regions 1517-1587. In the original Article, the authors chose two residues (V1517 and P1797) according to their old model and proposed that mutations of these residues into cysteine would introduce a disulfide bond that could lock the $\mathrm{L}$ protein into a closed architecture. The results of surface plasmon resonance capping binding assays supported the abolishment of capping binding of this double mutant protein. In the re-refined model these two residues are $13 \AA$ apart, which is not likely to directly introduce the disulfide bond at their positions in the structure. The authors now reason that this double mutant might introduce a possible disulfide bond with significant conformational change to the SFTSV-L domains, as shown in their published work.

In light of these findings, the 'An Arg finger occupies the cap-binding pocket of the CBD' section of the Results has been revised. The sentence beginning 'We assume that mutations...' has been altered to read 'We assume that mutations into cysteine in the interface can introduce a disulfide bond and might lock SFTSV-L in this compact architecture.' In the same paragraph, the sentence beginning 'Consistent with our assumption...' has been altered to read 'Although P1797 in the CBD domain is $13 \AA$ away (Fig. 2f) from V1517 in the PB2-N-like domain, the SFTSV-L V1517C-P1797C mutant significantly abolished the binding of the m7G cap analogue in the in vitro cap-binding analysis assay (Fig. 2g,h)'. Finally, the sentence 'We reasoned that great conformational changes occurring in this double mutant may introduce the formation of a C1517-C1797 disulfide bond' has been inserted immediately following the revised sentence beginning 'Although P1797 in the....'

In addition, Fig. 2d-f has been corrected to show the revised structure of SFTSV-L and the interacting regions in wild-type SFTSV-L and the V1517C-P1797C mutant. The original and corrected figures are shown below.

These modifications do not affect the authors' conclusions about the role of the Arg finger in capping binding. The authors also re-refined regions 1-32, 350-380 and 384-390 and confirm that improvements in these regions do not affect any other conclusions, figures or text.

Published online: 21 April 2021

https://doi.org/10.1038/s41564-021-00906-y

๑ The Author(s), under exclusive licence to Springer Nature Limited 2021 


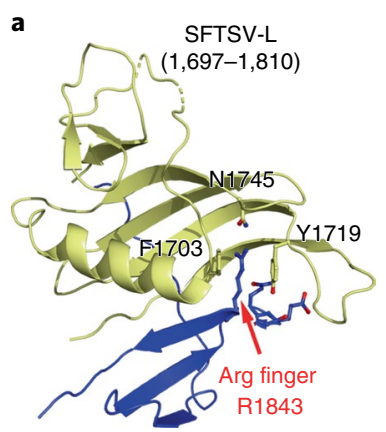

Original
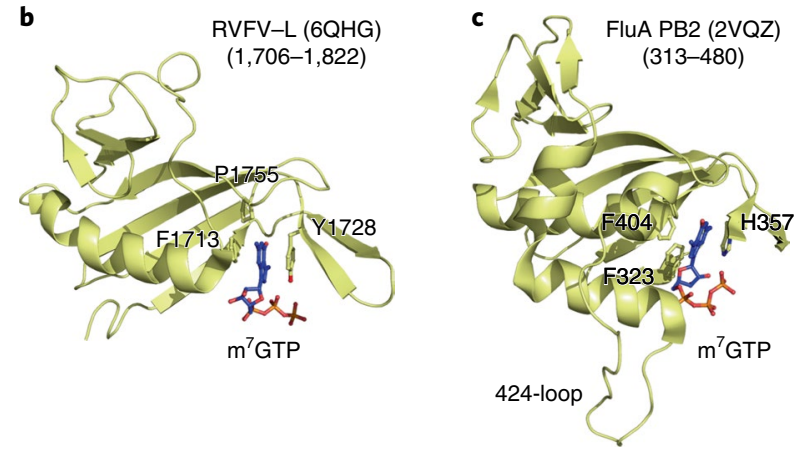

d

e
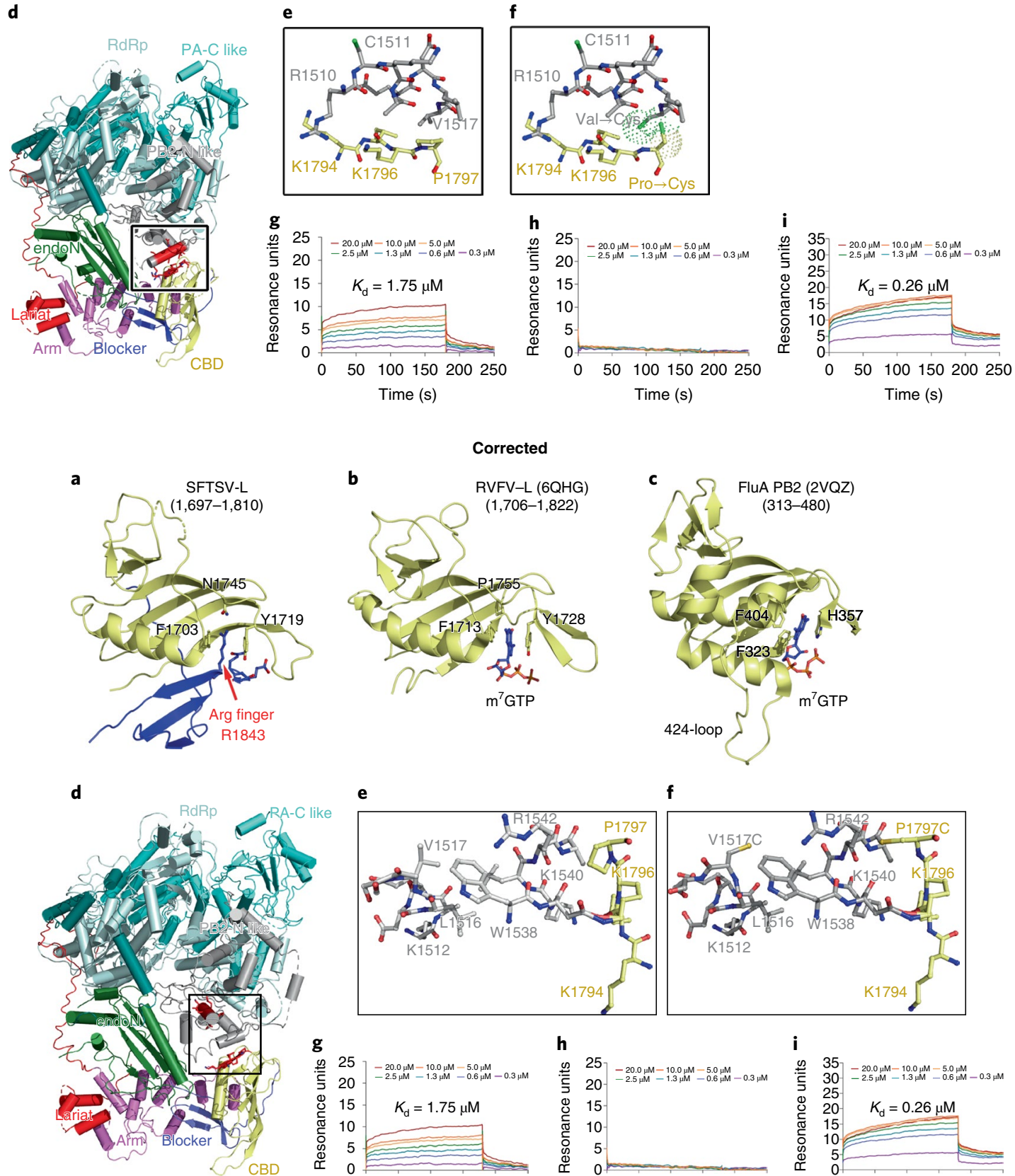

e

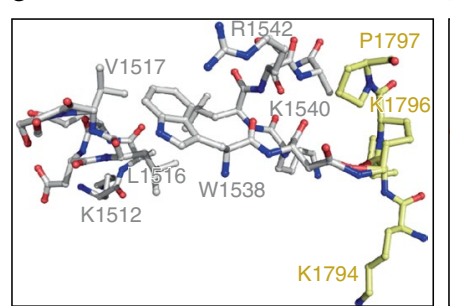

f
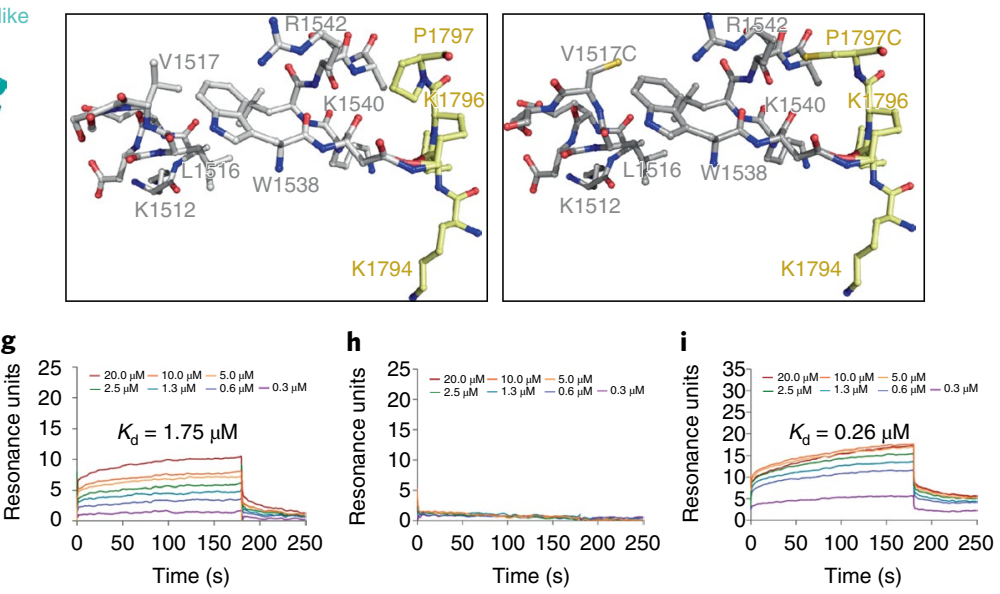

Fig. 2 | Original and Corrected. 